\title{
Exploiting Negative Impedance Converters to Extend the Bandwidth of LEDs for Visible Light Communication
}

\author{
Amany Kassem and Izzat Darwazeh \\ Department of Electronic and Electrical Engineering, University College London, London, United Kingdom \\ Email: amany.kassem.15@ucl.ac.uk, i.darwazeh@ucl.ac.uk
}

\begin{abstract}
This paper proposes, for the first time, a lossless light emitting diode (LEDs) bandwidth extension technique based on the adoption of negative impedance converters applied in the context of high capacity visible light communication systems. The proposed technique aims to achieve bandwidth extension by offsetting the bandwidth-limiting effect of the LED diffusion capacitance through the introduction of a parallel negative capacitance. With the proposed technique, we predict up to $200 \%$ improvement in the LED modulation bandwidth with studies based on a verified LED equivalent model.
\end{abstract}

Index Terms - Light emitting diodes, visible light communication, negative impedance converters, negative capacitance.

\section{INTRODUCTION}

Visible light communication (VLC) is a growing technology that has attracted significant research attention in recent years $[1,2]$. VLC is enabled by intensity modulating the optical emitted power of LEDs in the visible light range of the electromagnetic spectrum. Hence, LEDs serve the dual functionality of illumination and communication.

The major challenge facing VLC systems is in improving data transmission speeds, considering the low modulation bandwidth of commercially available LEDs, typically limited to few mega-Hertz [1-3]. The generation of white light by LEDs is predominately achieved using a single blue indium gallium nitride (InGaN) LED with a complementary colour converting material such as yellow phosphor (Ce:YAG). The LED bandwidth is mainly limited by the carrier lifetime and the RC time constant associated with the dynamic resistance $\left(R_{c}\right)$ and the diffusion capacitance $\left(C_{c}\right)$ of the LED; and the slow response of the colour converting material $[1,4,5]$.

To overcome the LED bandwidth limitations, there have been proposals of using blue optical filters to undo the effect of the slow yellow component by isolating the fast blue component which can extend the LED bandwidth up to $20 \mathrm{MHz}$ [6]. However, this approach results in significant reduction in the signal power and hence, reduced signal-tonoise-ratio (SNR). Moreover, the bandwidth of the blue LED, limited to 20-30 MHz still remains a possible bottleneck for high speed data communication [2]. Other approaches aimed to extend the LED bandwidth by utilising passive high pass filters to equalise different regions of the LED response [7]. Whereas, others have resorted to the use of efficient driver circuitry that reduces the optical pulse fall time as in $[7,8]$.
More recently, the adoption of artificial transmission lines attempted to incorporate the LED diffusion capacitance into a pseudo-artificial transmission line [5]. While most of the aforementioned approaches have been effective in achieving high data rates VLC links, their benefits may be debatable since they often incur high optical power penalties. Hence, limiting the LED output light intensity and possibly the overall system capacity due to the significant reduction in the SNR. Alternatively, the use of digital equalisation has been demonstrated to achieve reasonable transmission speeds without trading signal power [9] or alternatively adopting multi-carrier modulation techniques to effectively utilise the LED bandwidth as in [2], yet these techniques can significantly add to the system complexity.

An interesting circuit design concept is the realisation of negative impedance using active devices. The synthesis of negative impedance has been long seen as a viable approach to boosting the performance across a number of applications such as the enhancing the bandwidth of amplifiers [10,11], design of active filters [12] or non-Foster matching of electrically small antennas [13]. Negative impedance can be realised using networks known as negative impedance converters (NICs), originally proposed by Linvill in 1953 [14].

In this work, we propose, for the first time, a lossless LED bandwidth extension technique that is based on the adoption NICs. The idea is to introduce negative capacitance in parallel with the bandwidth-limiting LED diffusion capacitance, hence reducing the overall capacitance dictating the bandwidth. The proposed technique capitalises on the distinct characteristics of individual LEDs to achieve significant bandwidth extension without sacrificing the LED optical power.

\section{DESIGN CONCEPT}

The proposed LED bandwidth extension technique is studied by initially introducing the means by which the synthesis of negative capacitance can be practically realised through the design of a novel and simple current inversion NIC circuit. Moreover, the input impedance characteristics of the proposed NIC are analysed and a simple passive equivalent model emulating the NIC input impedance is derived.

Subsequently, we examine the LED impedance characteristics independently and when connected to the negative capacitance synthesised by the NIC through the analysis of 
simplified passive equivalent circuits. The LED impedance characteristics are particularly relevant to the study, as when driven by a current source the LED impedance exhibits similar frequency behaviour as the current $i_{d}$, which was shown in [5]. The effect of proposed technique is then demonstrated through simulation based on a verified equivalent LED model [4].

\section{A. Negative impedance converter design}

A NIC is defined as a two port network that presents negative impedance at one port when terminated by the corresponding load impedance at the other port as shown in Fig. 1, where $Z_{\text {in }}=-K Z_{L}$. The so-called conversion/ideality factor is given by $K$, which reflects quality of the generated negative impedance and unity $K$ indicates an ideal NIC.

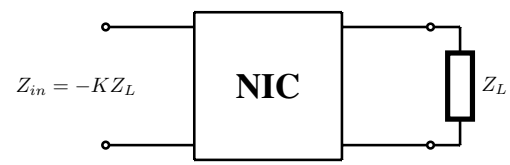

Fig. 1: Ideal 2-port representation of NIC

As stated by [15] negative impedance can be achieved by connecting the output of an amplifier, back to the input in series or shunt to form a positive feedback loop. The converter can be of a voltage inversion nature, where the input and output voltage is of opposite polarity. Alternatively, it can be of a current inversion nature where the current at one of the ports is inverted with respect to the other port. The NIC can be designed to generate either negative, resistance, capacitance and inductance depending on the terminating load impedance. Clearly here, the NIC is designed for capacitance due to the LED limitations.

Fig. 2 illustrates a practical implementation of a novel current inversion NIC consisting of a common collector stage $Q_{1}$ and a common base stage $Q_{2}$. The positive feedback is created by connecting the output and input of the common collector stage through a low input impedance common base stage $Q_{2}$. Hence, the load impedance $C_{c}$ between the emitters of $Q_{1}$ and $Q_{2}$ is negated at the input.

The NIC input impedance is obtained by applying small signal analysis to Fig. 2, the analysis is conducted under the assumption that the transistors are ideal and can be modelled by a simple DC-circuit model where internal capacitances such as the base-emitter $\left(C_{\pi}\right)$ and base collector $\left(C_{\mu}\right)$ capacitance are set to zero. The assumption of the simplified model aims to obtain a compact NIC input impedance equation to be able to derive a passive circuit equivalence, yet without affecting the accuracy at which the NIC input impedance is modelled. Hence, the NIC input impedance, resistance and reactance are given by:

$$
\begin{gathered}
Z_{i n} \approx \frac{g_{m 2}\left(1+g_{m 1} r_{\pi 1}\right)+j \omega c_{c}\left(1+g_{m 1} r_{\pi 1}+g_{m 2} r_{\pi 1}\right)}{\left(j \omega c_{c} r_{\pi 1} g_{m 1} g_{m 2}\right)} \\
R_{c} \approx-\frac{g_{m 1}+g_{m 2}}{g_{m 1} g_{m 2}} \\
X_{c} \approx \frac{1}{\omega C_{c}}
\end{gathered}
$$

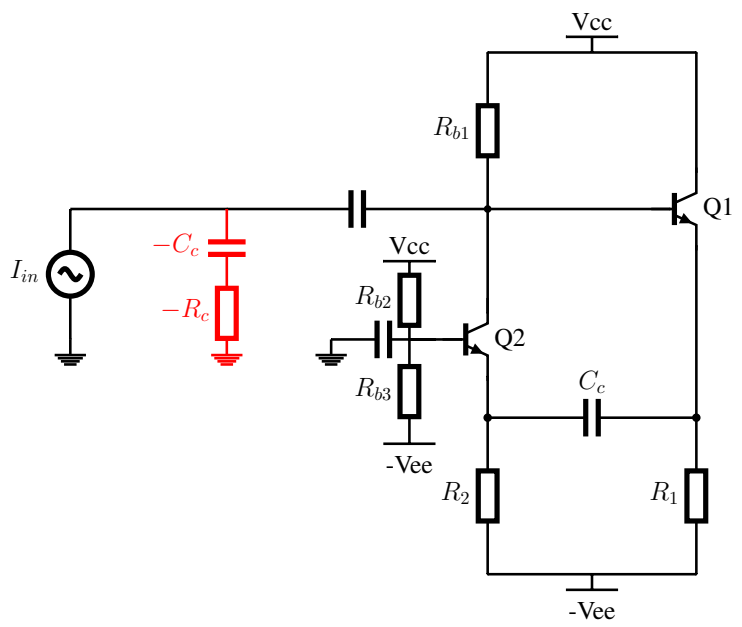

Fig. 2: Practical implementation of a current inversion NIC

By inspecting the derived equations for the NIC input impedance components (2) and (3), the following conclusions can be drawn: The NIC circuit presents an input impedance that is a combination of negative capacitance and negative resistance where the negative capacitance is equivalent to the terminating load capacitance $C_{c}$ and the negative resistance component is a function of the two transistor $Q_{1}$ and $Q_{2}$ bias currents. Moreover, it was found that the NIC input impedance is equivalent to these two components in series as shown by Fig. 2 (RED components).

The value of the negative resistance is particularly important as it dictates the quality of the negative capacitance generated. Ideally, this negative resistance component should be zero to obtain pure negative capacitance. However, it is practically impossible since it is a function transistors bias currents. Nevertheless, increasing the transistors bias currents would reduce the negative resistance, hence, enhancing the quality of the negative capacitance generated.

\section{B. Passive equivalent circuit analysis}

Fig. 3 illustrates a passive equivalent circuit of the wellknown LED model. The simplified LED model consists of the diffusion capacitance $C_{d}$, the small signal dynamic resistance $R_{d}$ and the Ohmic contact resistance $R_{s}$. By analysing the circuit in Fig. 3 the LED impedance is given by:

$$
Z_{i n}=R_{s}+\frac{R_{d}}{1+j \omega R_{d} C_{d}}
$$

The LED is effectively a first order low pass filter where at a fixed bias the bandwidth may be obtained by a time constant associated with $C_{d}$ and $R_{d}$.

It is possible to extend the LED bandwidth by ameliorating the bandwidth-limiting effect of $C_{d}$ by introducing a parallel negative capacitance $C_{c}$, therefore reducing the net capacitance dictating the bandwidth. Under ideal conditions, the bandwidth can be infinitely increased if $C_{c}$ is equal to the $C_{d}$, such that the LED bandwidth becomes:

$$
f_{3 \mathrm{~dB}}=\frac{1}{2 \pi R_{d}\left(C_{d}-C_{c}\right)}
$$




$$
Z_{\text {in }}=\frac{\left(R_{d} R_{s} C_{s} s+R_{d}+R_{s}\right)\left(R_{c} C_{c} s+1\right)}{\left(R_{d} C_{d} C_{c}\left(R_{s}-R_{c}\right)\right) s^{2}+\left(R_{d}\left(C_{d}-C_{c}\right)-C_{c}\left(R_{s}-R_{c}\right)\right) s+1}
$$

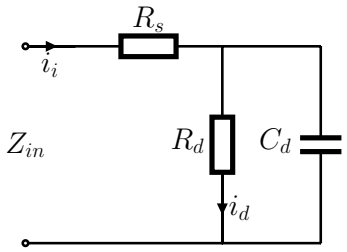

Fig. 3: Simplified LED equivalent circuit

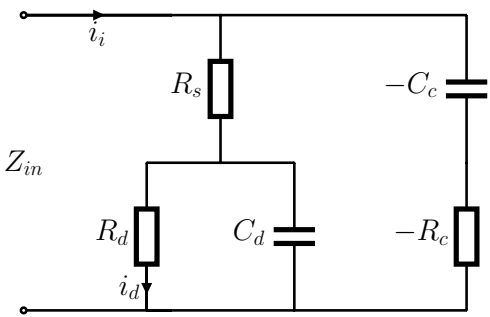

Fig. 4: Simplified LED equivalent in parallel with synthesised negative capacitance

However, it is not practically possible to realise a pure negative capacitance, yet the NIC presents an impedance combination consisting of $-C_{c}$ in series with $-R_{c}$ as shown by Fig. 4. By analysis of Fig. 4 the LED impedance becomes (6).

Where the DC resistance remains the same as in (4) i.e. $R_{d}+R_{s}$. Equation (6) is a transfer function that consists of two real zeros as shown in (7) and either a complex conjugate pole or a real pole. In cases were $R_{s}=\left|R_{c}\right|$, then the second order terms is cancelled hence, the transfer function has just one real pole as in (5).

$$
\omega_{z 1}=\frac{R_{s}+R_{d}}{R_{d} R_{s} C_{d}} \quad \omega_{z 2}=\frac{1}{R_{c} C_{c}}
$$

However, in cases where $R_{s} \neq\left|R_{c}\right|$, then the transfer function has a complex pole given by:

$$
p_{1,2}=-\frac{\omega_{n}}{2 Q}+j \omega_{n} \sqrt{1-Q^{2}}
$$

where $\omega_{n}$ is the complex conjugate pole natural frequency and $Q$ is the quality factor and are expressed by:

$$
\begin{gathered}
\omega_{n}=\frac{1}{\sqrt{R_{d} C_{d} C_{c}\left(R_{s}-R_{c}\right)}} \\
Q=\frac{\sqrt{R_{d} C_{d} C_{c}\left(R_{s}-R_{c}\right)}}{R_{d}\left(C_{d}-C_{c}\right)-C_{c}\left(R_{s}-R_{c}\right)}
\end{gathered}
$$

Hence, based on (6), three key observations can be made: (i), the LED bandwidth is no longer dependent on just $C_{d}$ and $R_{d}$. Moreover, the value of $R_{c}$ can be tailored based on the distinct characteristics of individual LEDs so that $R_{s}=\left|R_{c}\right|$ and hence, the LED bandwidth becomes dependent on $R_{d}$ and the net capacitance resulting from the difference between $C_{d}$ and $C_{c}$. Recalling, the value of $R_{c}$ is a function of the NIC bias current as previously described in (3). While, in practice a complete neutralisation of $C_{d}$ is difficult to achieve, maximum optimisation is possible. In cases where $R_{s} \neq\left|R_{c}\right|$ the system has a complex pole; the natural frequency of the complex pole $\omega_{n}$ increases as the value of the negative capacitance $C_{c}$ is increased (becomes more negative). As $\omega_{n}$ increases, it in turn shifts the frequency of $p_{1,2}$ to higher frequencies, thus extending the LED bandwidth. (ii) The frequency of the zero expressed in (7) is a function of $R_{c}$ and $C_{c}$.

Finally, (iii), the equivalent resistance of the LED when connected to the synthesised capacitance (NIC) is equal to the LED on its own. This mean that there will be no LED output power loss. In other words, the NIC circuit does not result in resistive loading. However, this is dependent on the exact design of the NIC. In case of the circuit in Fig 2, there will be additional loading due to the bias resistor $R_{b 1}$. However, if $R_{b 1}>>R_{s}+R_{d}$, such loading may be neglected. Hence, the proposed LED extension technique does not impose a bandwidth-power trade-off and the bandwidth gains does not come at the expense of optical power penalty as with techniques reported in [5].

\section{RESULTS}

In this section, we initially examine the quality of the negative capacitance $C_{c}$ generated by the NIC under different bias currents for transistors $Q 1$ and $Q 2$. The aim is to demonstrate the effect of $R_{c}$ on the quality of the $C_{c}$ generated by the NIC. The simulation of the NIC circuit utilises the BFR193 NPN silicon transistor terminated by $C_{c}=200 \mathrm{pF}$. Fig. 5 illustrates the values $C_{c}$ when $I_{b}=\{6,12$, and 25$\} \mathrm{mA}$. Clearly, it can be seen that increasing $i_{b}$ provides a more constant value of $C_{c}$, this is because as $i_{b}$ increases the value of $R_{c}$ is reduced as in 2. Hence, a higher quality $C_{c}$ is obtained.

Furthermore, we illustrate the effect of compensating for the bandwidth-limiting effect of the LED diffusion capacitance by exploiting NICs to generate negative capacitance in parallel with the LED. The simulation of the LED is based on the verified equivalent model [4] with the characteristic values derived at $2 \mathrm{~mA}$ drive current are $C_{d}=0.59 \mathrm{nF}, R_{d}=14.4 \Omega$ and $R_{s}=1.6 \Omega$.

Fig. 6 compares the LED impedance $Z_{i n}$ to three different cases at which the LED is connected to the NIC terminated by $C_{c}$ values of $200 \mathrm{pF}, 300 \mathrm{pF}$ and $400 \mathrm{pF}$, where the NIC is operated at $i_{b}=12 \mathrm{~mA}$. As reported in [4], the LED equivalent model is predicted to have a raw bandwidth of $18.7 \mathrm{MHz}$. When $C_{c}=200 \mathrm{pF}$, the NIC generates a negative capacitance of $-200 \mathrm{pF}$ which is in parallel to $C_{d}$, reducing the overall net capacitance. This results in approximately $76 \%$ improvement in the LED cut-off frequency, which is significant. As the 


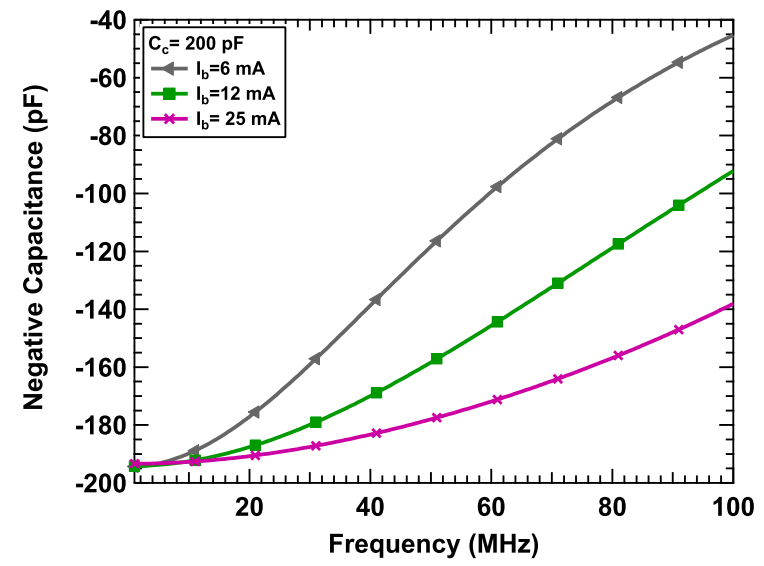

Fig. 5: Comparison of the $C_{c}$ generated by the NIC for different $I_{b}$

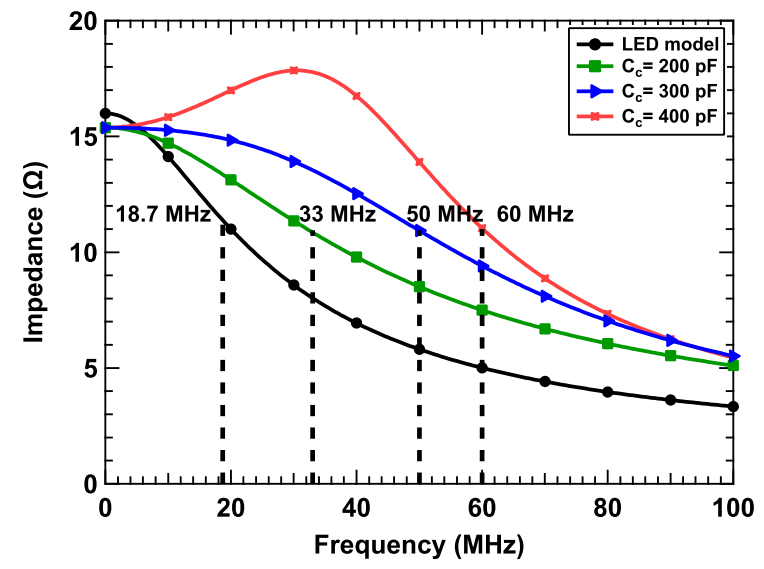

Fig. 6: Comparison of the LED impedance with different $C_{c}$

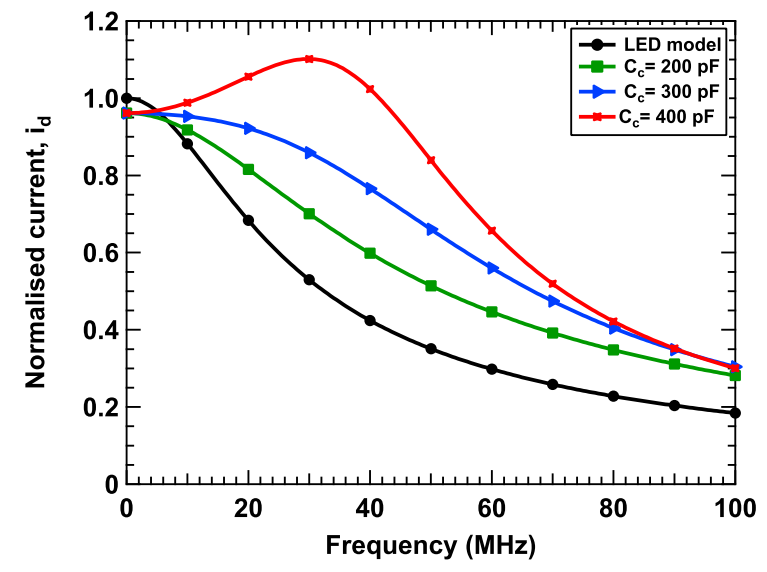

Fig. 7: Comparison of the LED current $i_{d}$ with different $C_{c}$

NIC load capacitance $C_{c}$ is further increased to $300 \mathrm{pF}$, the bandwidth increases by $167 \%$ in comparison to the model. Finally, in the case where $C_{c}=400 \mathrm{pF}$, the NIC is still effective in extending the bandwidth (a bandwidth improvement of $220 \%$ is obtained) yet a peak is observed in the response. The reason for this peak in the LED response is that the frequency of the zero in (7) is a function of the NIC load capacitance $C_{c}$.
Therefore, as $C_{c}$ increases the frequency of the zero shifts to lower frequencies and becomes much closer to the frequency of the complex poles which gradually perturbs the response as $C_{c}$ is increased further, resulting in peaking.

Fig. 7 presents the current $i_{d}$ through the LED for the same values of $C_{c}$. It is clear that the $i_{d}$ current exhibits the same frequency profile of the LED impedance for each of the $C_{c}$ cases. We notice that for increasing $C_{c}$ values, the current profile becomes flatter while maintaining the LED optical power until a critical point is reached as described previously. This is particularly significant since the gain in bandwidth extension does not come at the expense of loss in optical power as opposed to other reported techniques $[3,5,6]$.

\section{CONCLUSION}

In this paper, we have described a lossless LED bandwidth extension technique that introduces negative capacitance in parallel with the LED diffusion capacitance. The negative capacitance is achieved by exploiting a novel negative impedance converter circuit. Furthermore, a simplified passive equivalent model of the negative impedance presented by the NIC has been derived. We show that substantial bandwidth extension can be achieved by optimising the proposed NIC to generate high quality negative capacitance, while maintaining a constant the LED optical power.

\section{REFERENCES}

[1] P. H. Pathak et al., "Visible Light Communication, Networking, and Sensing: A Survey, Potential and Challenges," IEEE Commun. Surveys Tuts., 2015.

[2] S. Rajbhandari et al., "A review of gallium nitride LEDs for multigigabit-per-second visible light data communications," Semiconductor Science and Technology, 2017.

[3] H. L. Minh et al., "100-Mb/s NRZ Visible Light Communications Using a Postequalized White LED," IEEE Photon. Technol. Lett., 2009.

[4] W. N. Cheung, P. J. Edwards, and G. N. French, "Determination of LED equivalent circuits using network analyser measurements," in IEEE Conference on Optoelectronic and Microelectronic Materials and Devices, 1998.

[5] T. Odedeyi, P. A. Haigh, and I. Darwazeh, "Transmission Line Synthesis Approach to Extending the Bandwidth of LEDs for Visible Light Communication," in IEEE CSNDSP, 2018.

[6] J. Grubor et al., "Wireless High-Speed Data Transmission with Phosphorescent White-Light LEDs," in ECOC 2007, 2007.

[7] H. Tanaka, Y. Umeda, and O. Takyu, "High-speed LED driver for visible light communications with drawing-out of remaining carrier," in IEEE RWS 2011, 2011.

[8] H. Halbritter et al., "High-speed LED Driver for ns-Pulse switching of High-Current LEDs," IEEE Photon. Technol. Lett., 2014.

[9] P. A. Haigh et al., "Visible Light Communications: $170 \mathrm{Mb} / \mathrm{s}$ Using an Artificial Neural Network Equalizer in a Low Bandwidth White Light Configuration," J. Lightw. Technol., 2014.

[10] J. Han et al., "A Low-Power Gigabit CMOS Limiting Amplifier Using Negative Impedance Compensation and Its Application," IEEE Trans. Very Large Scale Integr. (VLSI) Syst., 2011.

[11] A. Rasekh and M. S. Bakhtiar, "Compensation Method for Multistage Opamps With High Capacitive Load Using Negative Capacitance," IEEE Trans. Circuits Syst. II: Exp. Briefs, 2016.

[12] J. G. Linvill, "RC Active Filters," Proceedings of the IRE, 1954

[13] S. E. Sussman-Fort and R. M. Rudish, "Non-foster Impedance Matching of Electrically-Small Antennas," IEEE Trans. Antennas Propag., 2009.

[14] J. G. Linvill, "Transistor Negative-Impedance Converters," Proceedings of the IRE, 1953.

[15] J. L. Merrill, "Theory of the negative impedance converter," The Bell System Technical Journal, 1951. 